\title{
Review
}

\author{
Anna Carobene*, Aasne K. Aarsand, William A. Bartlett, Abdurrahman Coskun, \\ Jorge Diaz-Garzon, Pilar Fernandez-Calle, Elena Guerra, Niels Jonker, Massimo Locatelli, \\ Mario Plebani, Sverre Sandberg and Ferruccio Ceriotti
}

\section{The European Biological Variation Study (EuBIVAS): a summary report}

https://doi.org/10.1515/cclm-2021-0370

Received March 26, 2021; accepted May 14, 2021;

published online May 31, 2021

\begin{abstract}
Biological variation (BV) data have many important applications in laboratory medicine. Concerns about quality of published BV data led the European Federation of Clinical Chemistry and Laboratory Medicine (EFLM) 1st Strategic Conference to indicate need for new studies to generate BV estimates of required quality. In response, the EFLM Working Group on BV delivered the multicenter European Biological Variation Study
\end{abstract}

*Corresponding author: Anna Carobene, Laboratory Medicine, IRCCS San Raffaele Scientific Institute, Via Olgettina 60, 20132 Milan, Italy, Phone: +39 02 26432850, E-mail: carobene.anna@hsr.it

Aasne K. Aarsand, Department of Medical Biochemistry and Pharmacology, Haukeland University Hospital, Bergen, Norway; and Norwegian Organization for Quality Improvement of Laboratory Examinations (Noklus), Haraldsplass Deaconess Hospital, Bergen, Norway

William A. Bartlett, School of Medicine, University of Dundee, Scotland, UK

Abdurrahman Coskun, Acibadem Mehmet Ali Aydınlar University, School of Medicine, Istanbul, Turkey

Jorge Diaz-Garzon and Pilar Fernandez-Calle, Hospital Universitario La Paz, and Quality Analytical Commission of Spanish Society of Laboratory Medicine (SEQC ${ }^{\mathrm{ML}}$ ), Madrid, Spain

Elena Guerra and Massimo Locatelli, Laboratory Medicine, IRCCS San Raffaele Scientific Institute, Milan, Italy

Niels Jonker, Certe-Wilhelmina Ziekenhuis Assen, Europaweg-Zuid 1, Assen, The Netherlands

Mario Plebani, Department of Laboratory Medicine, University Hospital of Padova, Padova, Italy. https://orcid.org/0000-00020270-1711

Sverre Sandberg, Department of Medical Biochemistry and Pharmacology, Haukeland University Hospital, Bergen, Norway; Norwegian Organization for Quality Improvement of Laboratory Examinations (Noklus), Haraldsplass Deaconess Hospital, Bergen, Norway; and Department of Global Public Health and Primary Care, University of Bergen, Bergen, Norway

Ferruccio Ceriotti, Central Laboratory, Fondazione IRCCS Ca' Granda, Ospedale Maggiore Policlinico, Milan, Italy. https://orcid.org/00000002-0958-5354
(EuBIVAS). This review summarises the EuBIVAS and its outcomes. Serum/plasma samples were taken from 91 ostensibly healthy individuals for 10 consecutive weeks at 6 European centres. Analysis was performed by Siemens ADVIA 2400 (clinical chemistry), Cobas Roche 8000, c702 and e801 (proteins and tumor markers/hormones respectively), ACL Top 750 (coagulation parameters), and IDS iSYS or DiaSorin Liaison (bone biomarkers). A strict preanalytical and analytical protocol was applied. To determine BV estimates with 95\% CI, CV-ANOVA after analysis of outliers, homogeneity and trend analysis or a Bayesian model was applied. EuBIVAS has so far delivered BV estimates for 80 different measurands. Estimates for 10 measurands (non-HDL cholesterol, S100- $\beta$ protein, neuron-specific enolase, soluble transferrin receptor, intact fibroblast growth-factor-23, uncarboxylated-unphosphorylated matrix-Gla protein, human epididymis protein-4, free, conjugated and \%free prostate-specific antigen), prior to EuBIVAS, have not been available. BV data for creatinine and troponin I were obtained using two analytical methods in each case. The EuBIVAS has delivered high-quality BV data for a wide range of measurands. The BV estimates are for many measurands lower than those previously reported, having an impact on the derived analytical performance specifications and reference change values.

Keywords: analytical performance specification; biological variation; EuBIVAS; reference change values.

\section{Background}

In 2014, the European Federation of Clinical Chemistry and Laboratory Medicine (EFLM) planned its 1st Strategic Conference entitled, "Defining analytical performance specifications 15 years after the Stockholm Conference” [1]. The Strategic Conference questioned whether the hierarchy of analytical performance specifications (APS) established in Stockholm [2] was still valid or in need of change [1, 3]. The conclusion was that a simplified hierarchy should be 
adopted comprising three models to define APS: the first model based on clinical outcomes, the second on biological variation (BV), and the third identified as state-of-theart [1]. The new hierarchy confirmed the significance of the BV model for setting the APS in laboratory medicine, for internal quality control [2] and for external quality assurance $[4,5]$.

In addition to defining APS, appropriately quantified and characterized BV data have many applications. The data can be used to establish reference change values (RCV) that enable objective assessment of significance of change in serial test results $[6,7]$, to estimate the number of samples required to calculate the homeostatic set point (NHSP) [7, 8], to assess the utility of conventional population-based reference intervals [9] and to derive personalized reference intervals (prRI) [10].

Different compiled sources of BV data for such applications have throughout the last decades been made available to the laboratory medicine community. In 1992, Fraser delivered a compilation of the BV data published between 1988 and 1991 [11]. A further significant compilation was published in 1999, by Carmen Ricos and colleagues within the Spanish Society of Clinical Biochemistry and Molecular Pathology (SEQC) [12]; this work produced a database that included BV data for more than 350 biochemical and hematological measurands that was subsequently made widely available via the Westgard website [13]. While these resources have proven to be useful, some questions have arisen about the quality of the constituent data sets made available. Presentations at the EFLM 1st Strategic Conference, and several published reviews, affirmed doubts about quality of BV data in current use, leading to the conclusion that the utility of published data may be compromised [14-18]. It has become clear that there are many factors which impact not only upon on the quality of the data, but also affect the application and transferability of BV data across populations and over time.

The Strategic Conference identified a need for critical appraisal of existing BV data, and a requirement for new studies to generate high-quality BV estimates. It was proposed that, ideally, a BV database should only include data sets from appropriately designed studies [3], and further recommended the delivery of a large multicenter study BV study to provide updated "high quality" BV estimates. This resulted in two significant EFLM initiatives. The first being development of the Biological Variation Data Critical Appraisal Checklist (BIVAC) [19], an instrument to assess whether existing published BV studies contain all elements necessary for the associated BV estimates to be considered fit for purpose and inclusion within the EFLM biological variation database [20]. The latter also enables publication of BV estimates based on meta-analysis of BIVAC compliant studies [19]. The second initiative resulted in the design and execution of the European Biological Variation Study (EuBIVAS) [21]. This project was undertaken by the EFLM working group on BV. It aimed to deliver highquality BV data using a multicenter approach that applied a strict BIVAC compliant protocol to enable establishment of a biobank of suitably characterized specimens for analysis.

EuBIVAS has generated a large amount of data, significantly updating the BV estimates with consequent impacts on the derived APS and other applications. The EuBIVAS project followed a stringent classical approach to generation and management of the data. Some limitations have come to light while delivering the study, suggesting the need for new approaches. The aims of this paper are to summarize the EuBIVAS outcomes, to discuss their implications in clinical practice, and to present and discuss some limitations found with the approach to estimate BV data.

\section{EuBIVAS design}

\section{Sample collection}

The project involved six European laboratories (Milan, Italy; Bergen, Norway; Madrid, Spain; Padua, Italy; Istanbul, Turkey; Assen, The Netherlands).

At the beginning of the study, 105 subjects were recruited. Three subjects were not included in the final cohort after application of the inclusion/exclusion criteria at the first collection, five people withdrew during the study for personal reasons.

Blood samples were collected from 97 volunteers (44 men, aged $20-60$ years; 43 women, aged $20-50$ years; 10 women, aged 55-69 years). Further exclusions from the final cohort were based on the laboratory measurements made at each visit. The health status and the inclusion/ exclusion criteria of the individuals enrolled in the EuBIVAS and the protocol used to collect, process, and store the samples have previously been reported in detail [21].

For each eligible individual, fasting blood samples were drawn weekly for 10 consecutive weeks (April-June 2015). A short questionnaire was completed, and a set of laboratory tests were performed at each sampling consisting of blood collected under controlled conditions to provide serum, $\mathrm{K}_{2}$ EDTA-plasma and citrated-plasma samples. A biobank of 18,000 aliquots was established 
consisting of 120 aliquots of serum, 40 of EDTA plasma, and 40 of citrated-plasma from each subject.

The collection started in all centers between the 13th and the 16th week of 2015. Following centrifugation serum and plasma samples separated from the drawn blood were aliquoted and frozen locally at $-80^{\circ} \mathrm{C}$ and stored until the end of the study period.

Samples were sent from each contributing center, frozen in dry ice, to the coordinating center - San Raffaele Hospital in Milan, Italy - and stored at $-80{ }^{\circ} \mathrm{C}$ until analysis (June 2016-January 2018). The EuBIVAS protocol was approved by the Institutional Ethical Review Board of San Raffaele Hospital in agreement with the World Medical Association Declaration of Helsinki and by the Ethical Board/Regional Ethics Committee for each center. All the participants signed informed consent.

\section{Analytical methods}

The list of analyzed measurands, instruments and reagents used are detailed in Supplementary Table 1. All analyses were calibrated according to the manufacturers' instructions. Further details are available in EuBIVAS publications [22-37]. For all measurands, all samples from the same study participant were analyzed in duplicate within a single run.

\section{Data analysis}

Data analyses were performed as previously described [21-23]. Briefly, within-subject $\mathrm{BV}\left(\mathrm{CV}_{\mathrm{I}}\right)$ estimates for all participants, males, females, and for other subgroups if considered relevant for specific measurands, were for most EuBIVAS measurands estimated by the Røraas method; which is based on a CV-ANOVA approach [38].

In addition, to estimate BV for 25-hydroxy vitamin D (25(OH)D), the results were also transformed into multiples of the median (MoM), and then the natural logarithm of MoM (lnMoM) was calculated [39]. The MoM transformation was applied to create a steady-state situation of the 25(OH)D parameters [34].

The Røraas method requires, prior to CV-ANOVA analysis, assessment for outliers for both replicates and samples on the CV-transformed data, and homogeneity of analytical $\mathrm{CV}\left(\mathrm{CV}_{\mathrm{A}}\right)$ and $\mathrm{CV}_{\mathrm{I}}$, examined by the Bartlett and Cochran tests, respectively. Trend analysis was performed to ensure steady state. When the individuals were not in steady state, data were adjusted according to the observed change.
$\mathrm{CV}_{\mathrm{I}}$ and between-subject $\mathrm{BV}\left(\mathrm{CV}_{\mathrm{G}}\right)$ estimates were calculated for male and female subgroups for all measurands. When visual inspection of data indicated differences in concentrations depending on age (i.e. females in fertile/ menopausal age, males $<30$ years and $>30$ years of age for prostate-specific antigen (PSA) [25]), or depending on the country of origin (like for creatinine [24], thyroid hormones [35]), BV estimates were also calculated for these subgroups accordingly.

$\mathrm{CV}_{\mathrm{G}}$ estimates were calculated on natural logtransformed data after assessment and elimination of outliers between individuals (Dixon criterion). When significant differences were observed between $\mathrm{CV}_{\mathrm{I}}$ of men and women, separate APSs were calculated. If mean concentrations between men and women were significantly different (lack of overlap of $95 \% \mathrm{CIs}$ ), the lower of the $2 \mathrm{CV}_{\mathrm{G}}$ was applied in the APS. Reference change values (RCVs) were calculated using the log normal approach [27, 28]. To deliver BV estimates for coagulation parameters [37], a Bayesian approach, as described by Røraas et al. [40], was applied.

Data analyses for CV-ANOVA were performed using Microsoft Excel 2010 and IBM SPSS statistics, version 23.

\section{EuBIVAS population}

Samples from six out of the 97 enrolled subjects were excluded for measurands analyzed by CV-ANOVA as one or more laboratory measurements in these subjects were outside the normal reference or action limits [21]. Two males were excluded on suspicion of subclinical viral infection based upon a significant negative trend in $y$-glutamyl transferase (GGT) and alanine amino transferase (ALT) values, 2 additional males were excluded because of elevated creatine kinase (CK)/ALT on a number of occasions, a fifth male with intermittent elevation of ALT, suspected of having liver disease, was excluded, while the 6th exhibited elevated ALT (three collections), CK (one pathological value) and C-reactive protein (CRP) concentrations (three pathological values) [21]. The characteristics of the EuBIVAS population (91 subjects, 38 men, 43 women in fertile age, and 10 women above 50 years, identified as menopausal) included in the CV-ANOVA studies are shown Table 1.

\section{BV estimates EuBIVAS based}

In Table 2, an overview of published EuBIVAS based BV estimates for, to date, 80 different measurands, is provided 
Table 1: Gender, number, age, and body max index (BMI) of men, women < 50, and women > 50 years enrolled by each center.

\begin{tabular}{|c|c|c|c|c|c|c|c|c|c|}
\hline & \multicolumn{3}{|c|}{ Men (20-60 years) } & \multicolumn{3}{|c|}{ Women (20-50 years) } & \multicolumn{3}{|c|}{ Women ( $50-70$ years) } \\
\hline & Number & $\begin{array}{r}\text { Age, years, } \\
\text { median and } \\
\text { range }\end{array}$ & $\begin{array}{r}\mathrm{BMI}, \mathrm{kg} / \mathrm{m}^{2}, \\
\text { median and } \\
\text { range }\end{array}$ & Number & $\begin{array}{r}\text { Age, years, } \\
\text { median and } \\
\text { range }\end{array}$ & $\begin{array}{r}\mathrm{BMI}, \mathrm{kg} / \mathrm{m}^{2}, \\
\text { median and } \\
\text { range }\end{array}$ & Number & $\begin{array}{r}\text { Age, years, } \\
\text { median and } \\
\text { range }\end{array}$ & $\begin{array}{r}\mathrm{BMI}, \mathrm{kg} / \mathrm{m}^{2}, \\
\text { median and } \\
\text { range }\end{array}$ \\
\hline $\begin{array}{l}\text { Italy-Milan (19 } \\
\text { persons) }\end{array}$ & 9 & $38(24-59)$ & $\begin{array}{r}25.2 \\
(20.8-30.0)\end{array}$ & 7 & $34(24-48)$ & $\begin{array}{r}22.7 \\
(17.6-23.9)\end{array}$ & 3 & $58(55-59)$ & $\begin{array}{r}22.8 \\
(19.4-27.5)\end{array}$ \\
\hline $\begin{array}{l}\text { Norway (15 } \\
\text { persons) }\end{array}$ & 7 & $37(28-42)$ & $\begin{array}{r}24.3 \\
(18.1-26.3)\end{array}$ & 6 & $39(29-49)$ & $\begin{array}{r}21.7 \\
(18.7-24.4)\end{array}$ & 2 & 63 & $\begin{array}{r}24.6 \\
(23.7-25.5)\end{array}$ \\
\hline $\begin{array}{l}\text { Spain (16 } \\
\text { persons) }\end{array}$ & 7 & $34(26-54)$ & $\begin{array}{r}25.1 \\
(19.5-32.5)\end{array}$ & 7 & $26(24-48)$ & $\begin{array}{r}21.7 \\
(17.9-23.1)\end{array}$ & 2 & 60 & $\begin{array}{r}21.3 \\
(21.2-21.4)\end{array}$ \\
\hline $\begin{array}{l}\text { Italy-Padua (14 } \\
\text { persons) }\end{array}$ & 5 & $32(27-35)$ & $\begin{array}{r}22.5 \\
(19.0-23.5)\end{array}$ & 8 & $33(27-49)$ & $\begin{array}{r}19.8 \\
(18.7-23.2)\end{array}$ & 1 & 69 & 18.6 \\
\hline $\begin{array}{l}\text { Turkey (15 } \\
\text { persons) }\end{array}$ & 6 & $27(22-35)$ & $\begin{array}{r}27.5 \\
(22.2-29.9)\end{array}$ & 9 & $33(21-38)$ & $\begin{array}{r}21.1 \\
(18.3-27.3)\end{array}$ & - & - & \\
\hline $\begin{array}{l}\text { The Netherlands } \\
\text { (12 persons) }\end{array}$ & 4 & $36(23-45)$ & $\begin{array}{r}24.0 \\
(18.1-26.3)\end{array}$ & 6 & $39(29-49)$ & $\begin{array}{r}21.7 \\
(20.9-24.2)\end{array}$ & 2 & $60(59-60)$ & $\begin{array}{r}23.0 \\
(20.7-25.3)\end{array}$ \\
\hline $\begin{array}{l}\text { Total (91 } \\
\text { persons) }\end{array}$ & 38 & $35(22-59)$ & $\begin{array}{r}24.4 \\
(18.1-32.5)\end{array}$ & 43 & $34(21-49)$ & $\begin{array}{r}21.3 \\
(17.6-27.3)\end{array}$ & 10 & $60(55-69)$ & $\begin{array}{r}22.1 \\
(18.6-27.5)\end{array}$ \\
\hline
\end{tabular}

[22-37]. For creatinine (Crea) [24] and troponin I (TnI) [28], $\mathrm{BV}$ estimates were obtained by measurements performed using two different methods, Jaffe/enzymatic and Siemens/Singulex respectively, and both datasets are shown in Table 2.

\section{EuBIVAS outcomes}

The EuBIVAS was designed and executed in compliance with EFLM recommendations for BV studies [19, 41]. The pre-analytical and analytical phases of the investigations were rigorously controlled [21]. For most of the included measurands, the confidence interval of the mean values overlapped between the different subgroups at each of the six study centers. Thus, there is no indication for any differences in pre-analytical variables or treatment between the different centres [42]. An exception is for serum creatinine, for which lower mean concentrations were observed in the Turkish cohort. This difference has been attributed to population differences, such as a higher body mass index (BMI) and the number of smokers in the Turkish cohort [24]. Differences between participants from different countries were also found for thyroid hormones, probably due to lifestyle differences and/or other factors linked to the ethnicity [35].

The EuBIVAS approach presents a number of benefits. Firstly, it is sufficiently powered [43] to enable subgroup analysis. This has allowed gender specific data which until this point been unavailable for many measurands.
Secondly, the BV estimates have been obtained based on current best-practice recommendations for study design $[19,41]$. The EuBIVAS estimates are therefore well characterized and documented, providing a level of understanding and confidence around the data to potential users for application as reference data.

Systematic reviews recently published by the Task Group for the BV database [44-48], identified only three out of 61 evaluated papers as being fully BIVAC compliant i.e. eliciting the highest BIVAC grade, "A", for kidney related analytes [44], one out of 59 for lipid biomarkers of cardiovascular risk [45], one out of 47 for diabetes related analytes [46], six out of 16 for cardiac troponins [47] and four out of 49 for tumor markers. For the tumor markers, all A-graded publications were derived from the EuBIVAS [48]. The EuBIVAS has been designed to fulfill the BIVAC criteria and is thus fully compliant with all the 14 BIVAC quality items, eliciting a BIVAC grade A [19].

The BIVAC grading is utilized in the meta-analysis of $\mathrm{BV}$ data that has been developed to deliver global estimates of BV that combines data from several studies and which are published in the EFLM BV database. In the metaanalysis approach, the combined result of the inverse width of the CI, which combines information from both the $\mathrm{CV}_{\mathrm{A}}$ and the number of subjects, samples, replicates, and the quality grade, decide the weight of each estimate [19]. Thus, for measurands for which EuBIVAS estimates represent a high percentage of the total weight in metaanalysis calculation, the EuBIVAS will have high influence on the meta-analysis results, such as if few papers are 


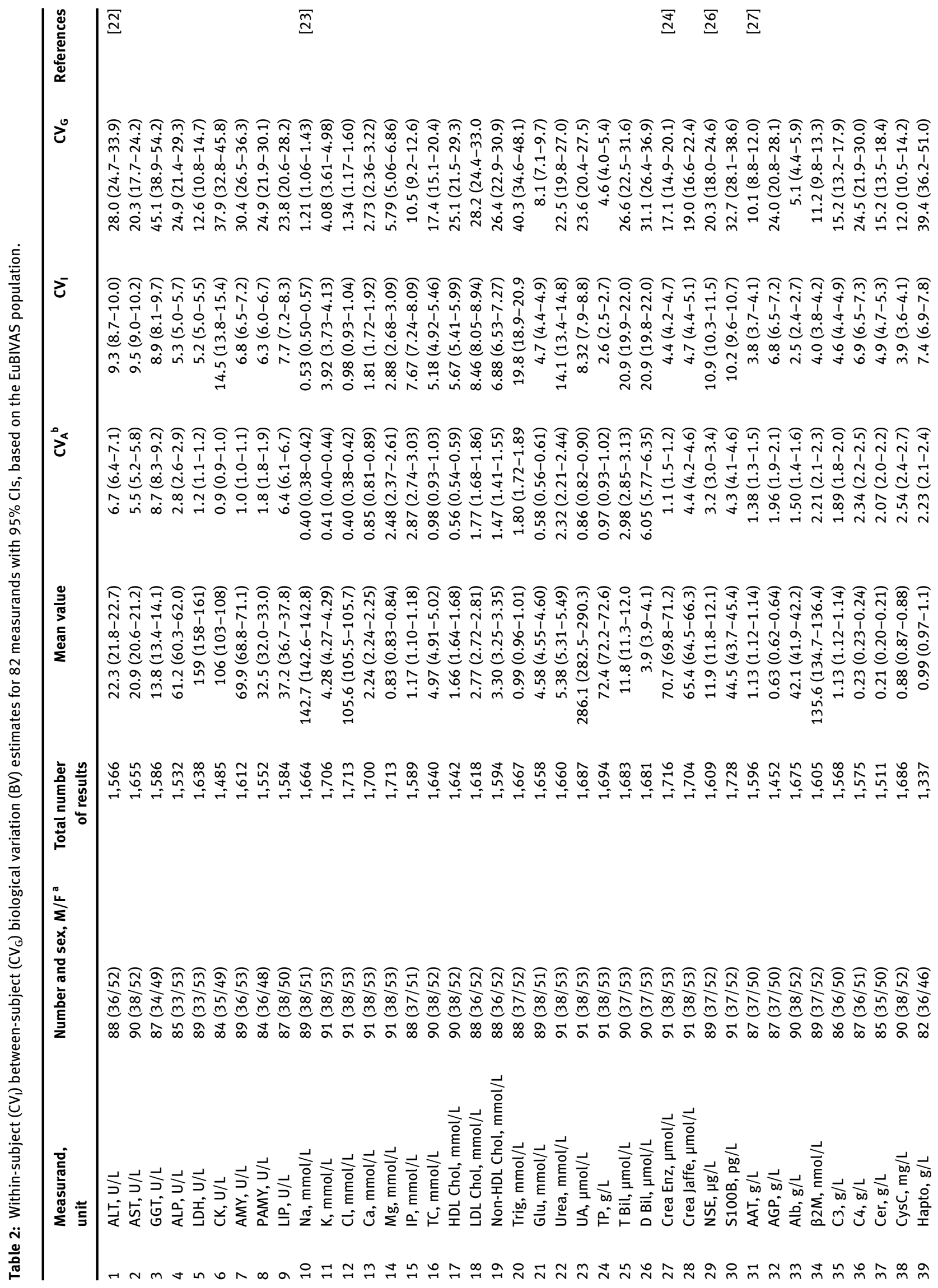




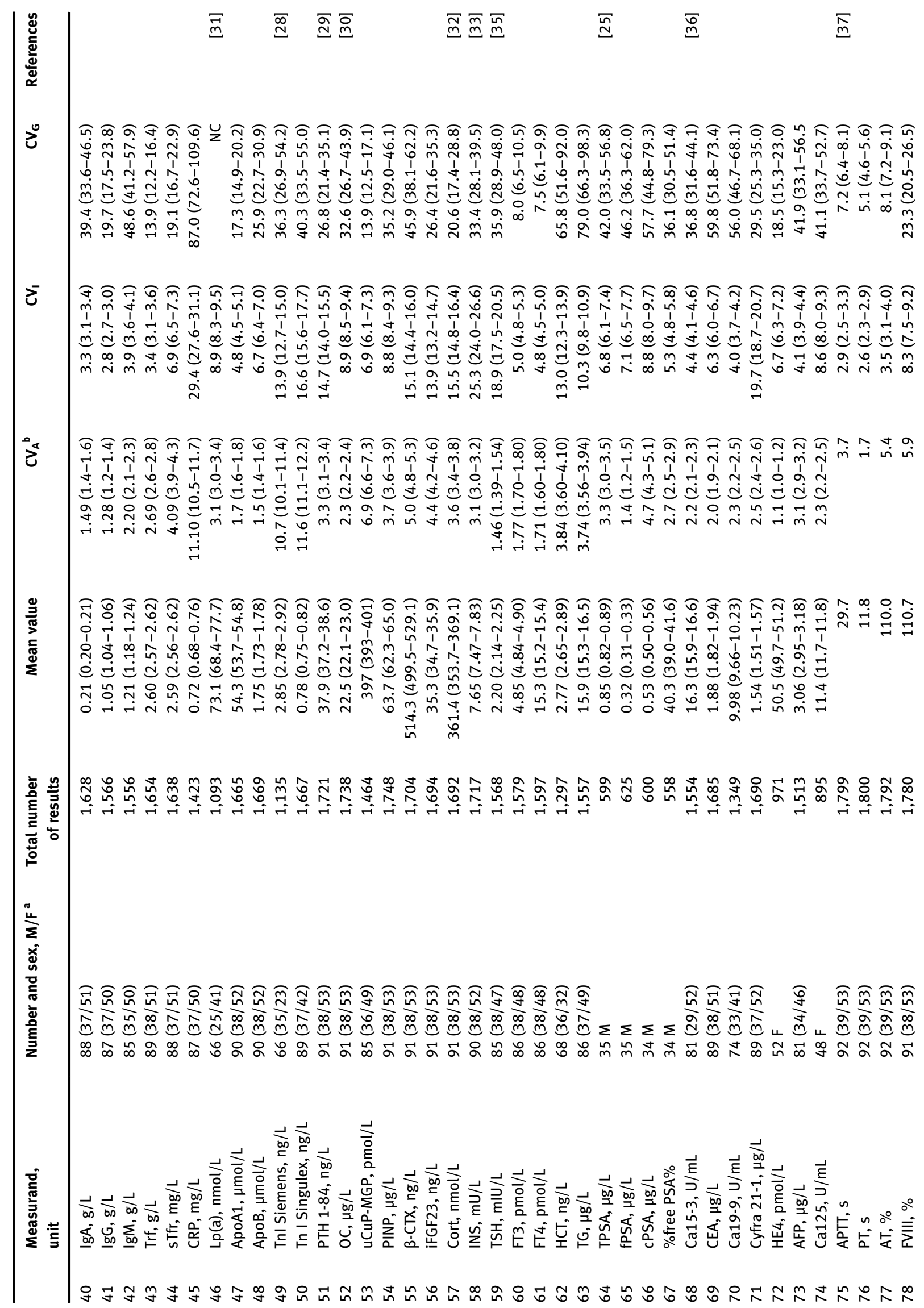




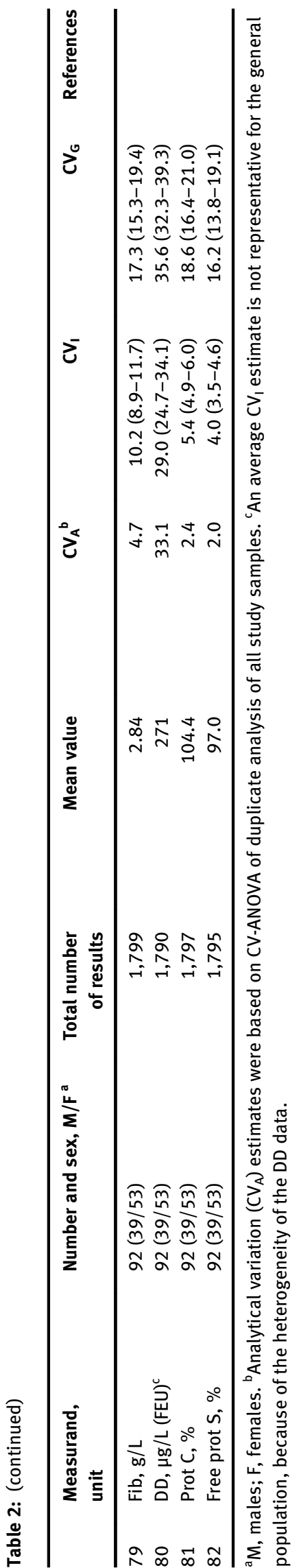

included in the meta-analysis, if none or a few of the included studies are given a BIVAC grade A, or if the included studies have a low number of subjects/samples.

EuBIVAS has so far delivered BV estimates for 80 different measurands, the results for which are presented in Table 2. Estimates for 10 measurands; non-HDL Cholesterol, S100- $\beta$ protein, neuron-specific enolase, soluble transferrin receptor, intact fibroblast growth factor 23 , uncarboxylated-unphosphorylated matrix-Gla protein, human epididymis protein 4 , free, conjugated and \%free prostate-specific antigen were previously not published and not featured in the historical online BV database hosted on the Westgard website [13].

Interestingly, the data indicate that for the most common measurands routinely measured in the clinical laboratories, EuBIVAS data did not really change the prior estimates, as displayed by Jonker et al. for albumin, creatinine, urea, chloride, sodium and potassium [44]. This is also demonstrable from the work of Díaz-Garzón et al. [45] for total, HDL and LDL cholesterol and for triglycerides, and by González-Lao et al. for glucose [46]. This is likely a result from the high number of BV studies performed for these measurands and in these cases, the highly-powered EuBIVAS provides a firm confirmation suggesting that additional general studies may not be warranted for these measurands. For other measurands, the EuBIVAS data has had major impact on the available estimates. Considering these findings, the high number of subjects and samples in the EuBIVAS and the "A" quality grade, it is beyond doubt that EuBIVAS estimates play an important role in defining the current global BV estimates and, consequently, in the definition of APS.

\section{Contribution of EuBIVAS outputs in the definition current global estimates: the example of BV estimates for enzymes}

For enzymes, most of the historical BV data was produced in 70 s and $80 \mathrm{~s}$, and derived using analytical methods now considered obsolete (not optimized according to the International Federation of Clinical Chemistry and Laboratory Medicine [IFCC]) or incompletely documented, being described as a "routine method" such as, for example, in $[16,22]$. Therefore, most papers (ranging from a minimum of 2 papers for pancreatic amylase and a maximum of 21 papers for aspartate amino transferase [AST]) were awarded the lowest quality grading "D" based on BIVAC quality item (QI) 4. QI4 relates to description of the measurand and to the measurement procedure. Moreover, the 
characteristics of the populations studied in the papers included in the historical estimates varied and include diseased (uncomplicated myocardial infarction, liver disease, type 1 diabetes mellitus) and non-diseased subjects $[12,16]$. As a consequence, very few historical BV studies of enzymes have been included in the metaanalysis delivered on the EFLM BV Database.

Table 3 shows EuBIVAS BV estimates for enzymes with 95\% CIs, accompanied by the corresponding BV estimates reported in the EFLM BV database [20], and the historical estimates published on the Westgard website [12]. For lactate dehydrogenase (LDH), amylase and pancreatic amylase, only the EuBIVAS enzyme publication has been considered appropriate for inclusion, after the exclusion of 15,9 , and 2 papers scored " $D$ " respectively. For CK and lipase, the global estimate is based on two publications, for ALT and AST three, and for GGT and phosphatase alkaline (ALP) four publications, respectively (Table 3).

The EuBIVAS is the only BV study for enzymes to have attained a grade " $A$ " by application of BIVAC (Table 3). Consequently, the global estimates reported in the EFLM database are very close to the EuBIVAS based estimates.

EuBIVAS estimates reporting lower BV estimates than those previously published produce stricter APS, used for both internal quality control and for external quality assurance (EQA). Generally, EQA providers should calculate their APSs in the same way [4] and use the EFLM BV database as their source for BV data. Thus, if EQA providers recalculate their APSs based on new and tighter data, it will be more difficult for participating laboratories to satisfy the new APS $[4,5]$.

EuBIVAS BV estimates produce not only stricter APS, but consequently also smaller RCVs, indicating that smaller changes between serial measurements are of significance than previously indicated when using RCVs derived from historical BV data [24, 25, 29]. In addition, resulting from lower $\mathrm{CV}_{\mathrm{I}}$ estimates, fewer samples are required to identify the NHSP in an individual $[25,35]$.

\section{Generalizability of the BV estimates}

\section{Significant differences between subgroups}

The second finding from EuBIVAS is that, for some measurands, significant differences between mean BV estimates were found in subgroups e.g., males/females; menopausal/fertile women; or for other occasional subgroups (e.g. for creatinine [24] and thyroid hormones [35], for which the country of origin was considered). To calculate $\mathrm{CV}_{\mathrm{G}}$, APS and RCV, the non-stratified BV estimate was used where no differences was found. If, however, stratification

Table 3: Biological variation (BV) estimates for EuBIVAS enzymes with $95 \% \mathrm{Cls}$, compared to the corresponding BV estimates reported in the EFLM BV database, and the historical estimates from the Westgard website. The number of papers included in the general estimate reported in the EFLM BV database is also indicated.

\begin{tabular}{|c|c|c|c|c|c|c|c|c|}
\hline \multirow[t]{2}{*}{ Enzyme } & \multicolumn{2}{|c|}{$\begin{array}{l}\text { EuBIVAS estimates } \\
\qquad(95 \% \mathrm{Cl})\end{array}$} & \multicolumn{2}{|c|}{$\begin{array}{c}\text { Global BV estimates from EFLM BV } \\
\text { database }^{a}\end{array}$} & \multirow[t]{2}{*}{$\begin{array}{l}\text { Number of } \\
\text { papers used } \\
\text { for the meta- } \\
\text { analysis and } \\
\text { BIVAC grade }\end{array}$} & \multicolumn{2}{|c|}{$\begin{array}{c}\text { Historical } \\
\text { values from } \\
\text { Westgard } \\
\text { website }\end{array}$} & \multirow[t]{2}{*}{$\begin{array}{l}\text { Number of } \\
\text { papers used to } \\
\text { derive the esti- } \\
\text { mates in West- } \\
\text { gard website }\end{array}$} \\
\hline & $\mathrm{CV}_{\mathbf{I}}$ & $\mathrm{CV}_{\mathrm{G}}$ & $\mathrm{CV}_{\mathbf{l}}$ & $\mathrm{CV}_{\mathrm{G}}$ & & $\mathrm{CV}_{\mathbf{I}}$ & $\mathrm{CV}_{\mathrm{G}}$ & \\
\hline $\mathrm{ALT}, \mathrm{U} / \mathrm{L}$ & $9.3(8.7-10.0)$ & $28.0(24.7-33.9)$ & $10.4(9.5-15.6)$ & $29.2(27.7-38.4)$ & $1 \mathrm{~A}, 2 \mathrm{C}$ & 19.4 & 41.6 & 9 \\
\hline AST, U/L & $9.5(9.0-10.2)$ & $20.3(17.7-24.2)$ & $9.5(9.4-13.5)$ & $20.4(19.8-23.8)$ & $1 \mathrm{~A}, 2 \mathrm{C}$ & 12.3 & 23.1 & 13 \\
\hline GGT, U/L & $8.9(8.1-9.7)$ & $45.1(38.9-54.2)$ & $8.8(7.4-12.0)$ & $41.3(30.9-41.8)$ & $1 \mathrm{~A}, 3 \mathrm{C}$ & 13.4 & 42.1 & 10 \\
\hline$A L P, U / L$ & $5.3(5.0-5.7)$ & $24.9(21.4-29.3)$ & $5.4(4.5-6.0)$ & $23.8(19.9-24.8)$ & $1 \mathrm{~A}, 3 \mathrm{C}$ & 6.45 & 26.1 & 22 \\
\hline $\mathrm{LDH}, \mathrm{U} / \mathrm{L}$ & $5.2(4.9-5.5)$ & $12.6(10.8-14.7)$ & $5.2(4.9-5.5)$ & $12.6(10.8-14.7)$ & $1 \mathrm{~A}$ & 8.6 & 14.7 & 11 \\
\hline $\mathrm{CK}, \mathrm{U} / \mathrm{L}$ & $14.5(13.8-15.4)$ & $37.9(32.8-45.8)$ & $16.0(15.4-29.5)$ & $31.8(25.7-41.5)$ & $1 \mathrm{~A}, 1 \mathrm{C}$ & 22.8 & 40 & \\
\hline AMY, U/L & $6.8(6.5-7.2)$ & $30.4(26.5-36.3)$ & $6.8(6.5-7.2)$ & $30.4(26.5-36.3)$ & $1 \mathrm{~A}$ & 8.7 & 28.3 & 7 \\
\hline PAMY, U/L & $6.3(6.0-6.7)$ & $24.9(21.9-30.1)$ & $6.3(6.0-6.7)$ & $24.9(21.9-30.1)$ & $1 \mathrm{~A}$ & 11.7 & 29.9 & 2 \\
\hline LIP, U/L & $7.7(7.2-8.3)$ & $23.8(20.6-28.2)$ & $9.2(7.7-41.1)$ & $24.8(23.1-37.6)$ & $1 \mathrm{~A}, 1 \mathrm{C}$ & 32.2 & 31.8 & 3 \\
\hline
\end{tabular}

ALT, alanine amino transferase; AST, aspartate amino transferase; GGT, $\gamma$-glutamyl transferase; ALP, alkaline phosphatase; LDH, lactate

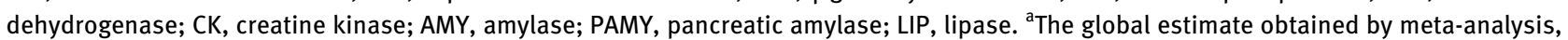
where the combined result of the inverse width of the $\mathrm{Cl}$ and the quality grade decide the weight of each estimate. When only one study is considered as fit to be included in the meta-analysis, the EFLM BV database reports as the lower and higher limits of the global estimate, the $95 \% \mathrm{Cl}$ derived from the included study. ${ }^{\mathrm{b}}$ The papers used to derive the general estimates reported in the Westgard website were not quality evaluated. 
into subgroups (e.g. age $<50$ years or sex) indicated that estimates were significantly different, the lowest, most stringent, BV estimate was applied to set APS. Estimates achieved in women $>50$ years were not used for APS calculations owing to the small number of participants $(n=10)$ in this group [23]. The small number of women in menopausal age enrolled in EuBIVAS represents an undoubted limitation of the study; if there are significant differences in a measurand between pre and postmenopausal women, the estimates obtained from this small subgroup may not be reliable enough to characterise this with confidence.

Gender differences have been also observed, for some measurands, in mean concentration distributions. Examples of gender differences can be observed in Figures 1 and 2 , where the distribution of mean concentrations for triglycerides (Trig), and urea, respectively, are represented in "Violin" plots. The distributions are accompanied with horizontal lines indicating the reference interval according to the manufacturer (Siemens Healthineers), for males (light blue) and females (pink) if different. Figures 1 and 2 show significant differences between gender for Trig and urea distributions, identified by difference in skew (Figure 1) or shapes (Figure 2). The fact that for these measurands, reference intervals are not traditionally stratified by sex may need to be revisited.

For many measurands (e.g. Trig, urea, uric acid, $\alpha 1$ acid glycoprotein (AGP), Immunoglobulin A (IgA), soluble

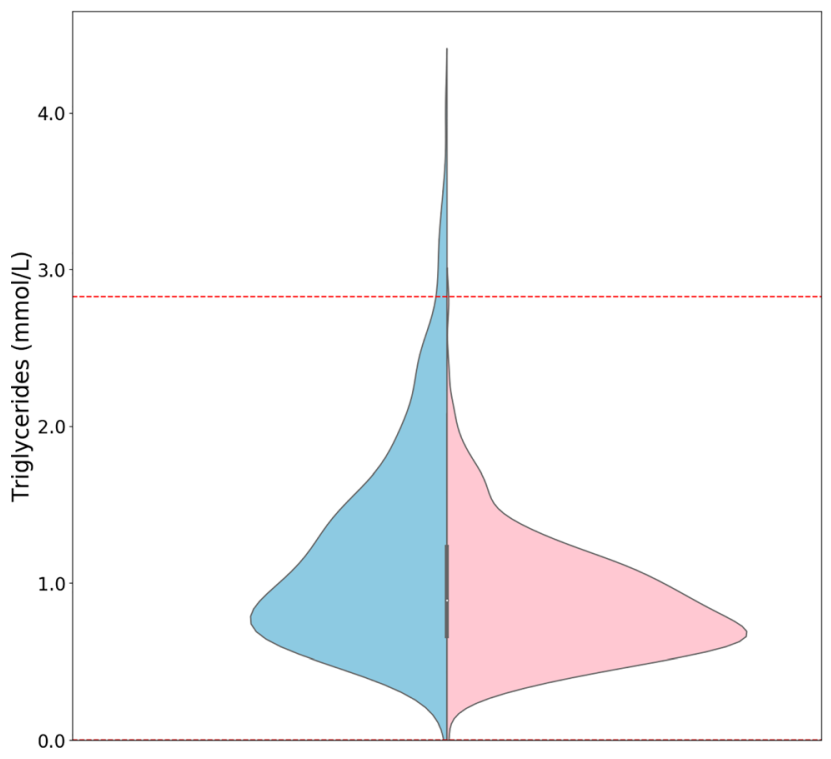

Figure 1: Violin plots depicting the distributions of mean values for triglycerides.

The light blue and pink areas correspond to males and females, respectively. The dashed lines indicate the reference intervals according to the manufacturer (Siemens).

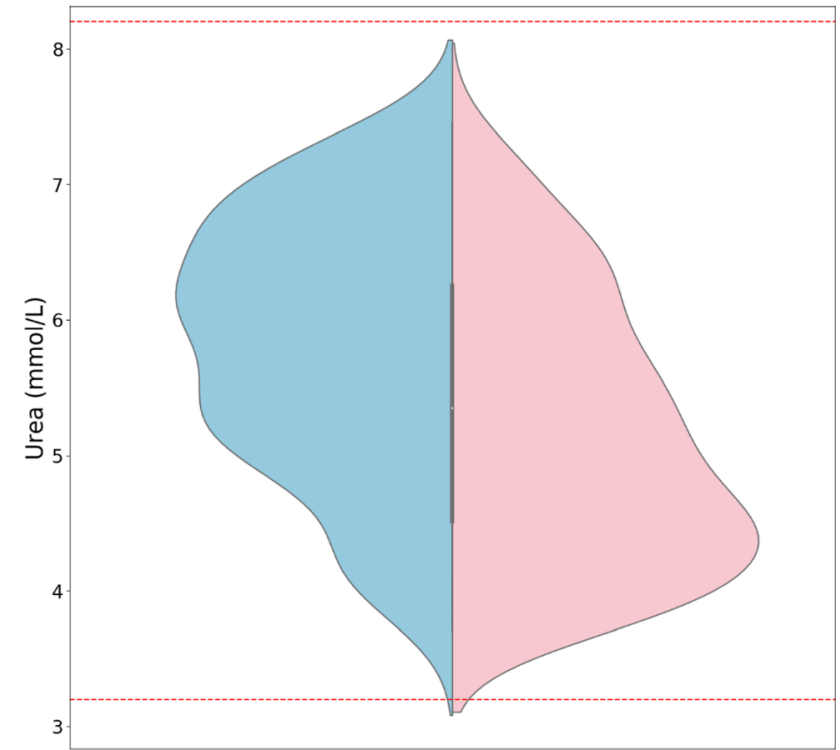

Figure 2: Violin plots depicting the distributions of mean values for urea.

The light blue and pink areas correspond to males and females, respectively. The dashed lines indicate the reference intervals according to the manufacturer (Siemens).

transferrin receptor (sTFR), lipoprotein(a) (Lp(a)), biointact parathyroid hormone (PTH 1-84), alpha fetoprotein (AFP), activated partial thromboplastin time (APTT), protein C (prot $\mathrm{C}$ ) and protein $\mathrm{S}$ free (free Prot $\mathrm{S}$ ), sex related differences between $\mathrm{CV}_{\mathrm{I}}$ were found $[23,27,29,31,36,37]$. Interestingly, with the only exception being for Trig, the lowest $\mathrm{CV}_{\mathrm{I}}$ was always observed in the male group. Moreover, when a significant difference was found also between females $<50$ and females of menopausal age, the $\mathrm{CV}_{\mathrm{I}}$ estimate for the menopausal women was more similar to the $\mathrm{CV}_{\mathrm{I}}$ of the male group. Even if from a statistical point of view, the differences are small, and while for some measurands it may be difficult to present a specific mechanism for this, hormonal differences would present a suitable starting point for investigation of causality. Generally, most available BV data are derived from healthy adults, and further studies are required to provide robust BV data for children, elderly and patients with relevant disease states.

\section{Steady state, the example of the inflammation marker proteins}

The classical experimental approach to estimate BV data is not necessarily the optimal approach for delivery of APS. As reported by Ceriotti et al. [49], there are limitations to the standard approach, including the need to carefully assess 
the relevance and validity of the BV data, e.g. the presence of 'steady state', the appropriateness of time intervals, effect of underlying illness and impact of measurand concentrations. According to Ceriotti et al. [49] the BV model for calculating APS is better applied for measurands that are under strict homeostatic control.

Most protein measurands of interest are not subject to strict homeostatic control. This is particularly true of commonly measured enzymes, whose serum concentrations derive from an equilibrium between production and elimination with many factors impacting each of these 2 modulators [22, 27]. A significant challenge in the evaluation of the BV estimates for specific proteins using EuBIVAS data, was the high number of samples with increased concentrations, caused by assumed acute phase episodes. If the CRP data are used as a potential marker of the presence of an acute phase episode, about $25 \%$ of participants had, at least, one episode of mild inflammation during the 10 weeks of sample collection [27]. This is the reason for a high rate of exclusion of results for a number the proteins investigated (from $4.9 \%$ for $\beta_{2}$-microglobulin ( $\beta 2 \mathrm{M}$ ) to $20 \%$ for haptoglobin). In some cases, exclusion of a high percentage of results, e.g., as for haptoglobin, might have limited the generalizability of the estimates as they may have caused an underestimation of the BV [27], which will have impact on their utility in applications such as determination of APS. Of the three models identified by the EFLM for determination of APS, it has been proposed that the "state of the art" is the best option to apply to CRP, to derive APS [50]. It is therefore likely that the same model should be used for other inflammatory marker proteins.

\section{Steady state, the example of the 25-hydroxy vitamin D (25(OH)D)}

The application of the classical model, the validity of which requires random variation of a measurand around homeostatic set point, turned out be inappropriate for the generation of BV estimates for the 25(OH)D [34]. The desirable statistical homogeneity of EuBIVAS cohort could only be achieved after elimination of more than $50 \%$ of the data. Examination of the data revealed that there was no steady state in 25(OH)D concentrations across time, a necessary pre-requisite for the BV estimates derivation [19, 41].

An alternative approach was adopted to identify a steady-state situation of the $25(\mathrm{OH})$ measurands. The EuBIVAS data set were analyzed after transformation to MoM, the approach suggested by Kristoffersen et al. [39], but also failed to deliver evidence of steady state, in that to achieve the necessary homogeneity of $\mathrm{CV}_{\mathrm{I}}$, more than $40 \%$ of data were eliminated. This was evidenced further by overt differences in the individual trends of each subject (data not shown). In consequence, the BV model cannot be used to set APS for 25(OH)D examinations. As in the case of acute phase proteins, an alternative approach to determine APS is required.

A proposal has been put forward by Cavalier et al. [34], that the probability of detection of $25(\mathrm{OH}) \mathrm{D}$ variation over a certain period of time could become the new paradigm to evaluate 25(OH)D examination methods. EuBIVAS results showed that, in a European population, $25(\mathrm{OH}) \mathrm{D}$ concentrations increased by $2.8 \%$ weekly over spring and that after 10 weeks, the mean increase was $31.6 \%$. The approach proposed by Cavalier et al. [34], is to set APS for measurement uncertainty (MU) based on the physiological variation of $25(\mathrm{OH}) \mathrm{D}$ concentrations over time. Accordingly, if following the proposed model, the higher order reference methods should present a MU $<1.2 \%$ and "routine" assays a MU $<13.6 \%$ to detect a difference (increase) at $\mathrm{p}<0.05$ and $9.6 \%$ to detect a difference at $\mathrm{p}<0.01$ [34].

It is of note that the model proposed by Cavalier applies only the setting of APS for the $25(\mathrm{OH}) \mathrm{D}$, which is only one of the applications of the BV estimates. To deliver the functionality of the many other applications (e.g. RCV, utility of reference intervals etc.) alternative statistical approaches should be sought.

\section{Concluding remarks}

The undertaking of studies of $\mathrm{BV}$ is challenging, and demanding of resource, particularly if they are to be delivered at scale and being fully compliant with BIVAC. The EuBIVAS multicenter approach has taken on this challenge and delivered data from a well characterized multinational population. The size and composition of the population studied enables stratification of the BV data sets by characteristics, such as age and sex with welldefined confidence limits. This makes the data generalisable and negates the need for local studies and the commitment of resource those would require.

It has been identified that the classical model for delivery of indices of biological variation is not suitable for all measurands for the setting of APS. This issue and others highlight the fact that rigorous multilevel examination of the raw data sets is a critical part of the classical model.

EuBIVAS has delivered generalisable high-quality BV data for a wide range of measurands, using samples collected under optimal conditions, minimizing preexamination sources of variation, using modern examination methodology and statistical analyses compliant 
with EFLM recommendations [19, 41]. The study delivers updates of historical BV data as well as data for new measurands. The EuBIVAS BV estimates are, for many measurands, lower than previously reported. This may be as a consequence of a combination of the rigor of the approach (subject selection, tight control of pre-analytical factors etc.), the use of modern examination methodology with higher specificity and the critically important application of correct statistical approach to data handling with exclusion of outliers [41, 51].

Finally, the absence of clear differences between groups from Turkey, Norway, The Netherlands, Spain, and Italy confirms that the obtained data are internationally transportable across healthcare systems and that they can be used to deliver APS for systems to be used internationally.

Acknowledgments: We would like to thank all the volunteers involved in the project. Without their contribution, the study could not have been realized. We thank the following co-workers who helped the authors in different steps of the project: Gerhard Barla, Beatriz Boned, Etienne Cavalier, Giuseppe Banfi, Michela Bottani, Fernando Marqués-García, Una Ørvim Sølvik, Marit Sverresdotter Sylte, Mustafa Serteser, Francesca Tosato, Christian Uccheddu, and Ibrahim Unsal. We thank Eric Kilpatrick, EFLM Science Committee Chair, for his continued support on repeated prompting. We are grateful to the Italian Society of Clinical Biochemistry and Clinical Molecular Biology (SIBioC) to the sponsorship that allowed the kick off of the project, and to Becton Dickinson, for donating us the blood collection equipment used by all centres. We also thank Siemens, Roche and Werfen for kindly providing us with the kits necessary for carrying out the study. We would like particularly to thank Eugene Harris, Callum Fraser G, Carmen Ricos and Per Hyltoft Petersen, the pioneers of the "biological variation" who inspired the EuBIVAS and who, in different ways, contributed to the project realization. We are in great debt to Thomas Røraas for his invaluable assistance in statistical analysis of the EuBIVAS data, and for having developed many of the new models used to calculate biological variation components and the metaanalysis that delivers global biological variation estimates in the EFLM Biological Variation Database.

Research funding: The Italian Society of Clinical Biochemistry and Clinical Molecular Biology (SIBioC) allowed the kick off of the project. Becton Dickinson, donated the blood collection equipment used by all centres. Siemens, Roche and Werfen provided the kits necessary for the measurements.
Author contributions: All authors have accepted responsibility for the entire content of this manuscript and approved its submission.

Competing interests: Authors state no conflict of interest. Informed consent: Informed consent was obtained from all individuals included in this study.

Ethical approval: The EuBIVAS protocol was approved by the Institutional Ethical Review board of San Raffaele Hospital in agreement with the World Medical Association Declaration of Helsinki and by the Ethical board/regional Ethics Committee for each involved center.

\section{References}

1. Sandberg S, Fraser GC, Horvath AR, Jansen R, Jones G, Oosterhuis $W$, et al. Defining analytical performance specifications: consensus statement from the 1st strategic conference of the European Federation of Clinical Chemistry and Laboratory Medicine. Clin Chem Lab Med 2015;53:833-5.

2. Fraser CG, Kallner A, Kenny D, Petersen PH. Introduction: strategies to set global quality specifications in laboratory medicine. Scand J Clin Lab Invest 1999;59:477-8.

3. Panteghini M, Sandberg S. Defining analytical performance specifications 15 years after the Stockholm conference. Clin Chem Lab Med 2015;53:829-32.

4. Carobene A, Franzini C, Ceriotti F. Comparison of the results from two different External Quality Assessment Schemes supports the utility of robust quality specifications. Clin Chem Lab Med 2011; 49:1143-9.

5. Haeckel R, Wosniok W, Kratochvila J, Carobene A. A pragmatic proposal for permissible limits in external quality assessment schemes with a compromise between biological variation and the state of the art. Clin Chem Lab Med 2012;50:833-9.

6. Fraser CG. Reference change values: the way forward in monitoring. Ann Clin Biochem 2009;46:264-5.

7. Fraser CG. The nature of biological variation. In: biological variation: from principles to practice. Washington, DC: AACC Press; 2001:1-27 pp.

8. Carobene A. La variabilità biologica: le basi teoriche e l'esperienza dei Gruppi di Lavoro della Federazione Europea di Chimica Clinica e Medicina di Laboratorio. Biochim Clin 2018;42: 15-25.

9. Fraser CG, Hyltoft Petersen P. Analytical performance characteristics should be judged against objective quality specifications. Clin Chem 1999;45:321-3.

10. Coskun A, Sandberg S, Unsal I, Cavusoglu C, Serteser M, Kilercik $M$, et al. Personalized reference intervals in laboratory medicine: a new model based on within-subjects biological variation. Clin Chem 2021;67:374-84.

11. Fraser CG. Biological variation in clinical chemistry. Arch Pathol Lab Med 1992;116:916-23.

12. Ricos C, Alvarez V, Cava F, Garcia-Lario JV, Hernandez A, Jimenez CV, et al. Current databases on biological variation: pros, cons and progress. Scand J Clin Lab Invest 1999;59:491-500. 
13. Ricos C, Alvarez V, Cava F, Garcia-Lario JV, Hernandez A, Jimenez CV, et al. Desirable specification for total error, imprecision, and bias, derived from intra- and inter-individual biologic variation. The 2014 update. Available from: www. westgard.com/biodatabase1.htm [Accessed Sept 2020].

14. Aarsand AK, Røraas T, Bartlett WA, Coşkun A, Carobene A, Fernandez-Calle $P$, et al. Harmonization initiatives in the generation, reporting and application of biological variation data. Clin Chem Lab Med 2018;56:1629-36.

15. Braga F, Panteghini M. Biological variability of $C$-reactive protein: is the available information reliable? Clin Chim Acta 2012;413 1179-83.

16. Carobene A, Braga F, Røraas T, Sandberg S, Bartlett WA. A systematic review of data on biological variation for alanine aminotransferase, aspartate aminotransferase and Y-glutamyl transferase. Clin Chem Lab Med 2013;51: 1997-2007.

17. Aarsand AK, Røraas T, Sandberg S. Biological variation - reliable data is essential. Clin Chem Lab Med 2015;53:153-4.

18. Carobene A. Reliability of biological variation data available in an online database: need for improvement. Clin Chem Lab Med 2015; 53:871-7.

19. Aarsand A, Roraas T, Fernandez-Calle P, Ricós C, Diaz-Garzon J, Jonker N, et al. On behalf of the EFLM Working Group on Biological Variation and Task and Finish Group for the Biological Variation Database. The biological variation data critical appraisal checklist (BIVAC): a new standard for evaluating studies on biological variation. Clin Chem 2018; 64:501-14.

20. Aarsand AK, Fernandez-Calle P, Webster C, Coskun A, Gonzales-Lao E, Diaz-Garzon J, et al. EFLM biological variation database. Available from: https://biologicalvariation.eu/ [Accessed Dec 2020].

21. Carobene A, Strollo M, Jonker N, Barla G, Bartlett WA, Sandberg S, et al. Sample collections from healthy volunteers for biological variation estimates' update: a new project undertaken by the Working Group on Biological Variation established by the European Federation of Clinical Chemistry and Laboratory Medicine. Clin Chem Lab Med 2016;54:1599-608.

22. Carobene A, Røraas T, Sølvik Uø, Sylte MS, Sandberg S, Guerra E, et al. Biological variation estimates obtained from 91 healthy study participants for 9 enzymes in serum. Clin Chem 2017;63: 1141-50.

23. Aarsand AK, Díaz-Garzón J, Fernandez-Calle P, Guerra E, Locatelli $M$, Bartlett WA, et al. The EuBIVAS: within- and between-subject biological variation data for electrolytes, lipids, urea, uric acid, total protein, total bilirubin, direct bilirubin, and glucose. Clin Chem 2018;64:1380-93.

24. Carobene A, Marino I, Coŝkun A, Serteser M, Unsal I, Guerra E, et al. The EuBIVAS project: within and between-subject biological variation data for serum creatinine using enzymatic and alkaline picrate methods and implications for monitoring. Clin Chem 2017;63:1527-36.

25. Carobene A, Guerra E, Locatelli M, Cucchiara V, Briganti A, Aarsand AK, et al. Biological variation estimates for prostate specific antigen from the European Biological Variation Study; consequences for diagnosis and monitoring of prostate cancer. Clin Chim Acta 2018;486:185-91.

26. Carobene A, Guerra E, Locatelli M, Ceriotti F, Sandberg S, Fernandez-Calle $\mathrm{P}$, et al. Providing correct estimates of biological variation-not an easy Task. The example of S100- $\beta$ protein and neuron-specific enolase. Clin Chem 2018;64:1537-9.

27. Carobene A, Aarsand AK, Guerra E, Bartlett WA, Coskun A, DíazGarzón Marco J, et al. European biological variation study (EuBIVAS): within- and between-subject biological variation data for 15 frequently measured proteins. Clin Chem 2019;65:1031-41.

28. Ceriotti F, Díaz-Garzón Marco J, Fernández-Calle P, Maregnani A, Aarsand AK, Coskun A, et al. The European Biological Variation Study (EuBIVAS): weekly biological variation of cardiac troponin I estimated by the use of two different high-sensitivity cardiac troponin I assays. Clin Chem Lab Med 2020;58:1741-7.

29. Bottani M, Banfi G, Guerra E, Locatelli M, Aarsand AK, Coşkun A, et al. European Biological Variation Study (EuBIVAS): within- and between-subject biological variation estimates for serum biointact parathyroid hormone based on weekly samplings from 91 healthy participants. Ann Transl Med 2020;8:855.

30. Cavalier E, Lukas P, Bottani M, Aarsand AK, Bartlett W, Ceriotti F, et al. European Biological Variation Study (EuBIVAS): within- and between-subject biological variation estimates of C-terminal telopeptides of type I collagen, $\mathrm{N}$-terminal propeptide of type I collagen, osteocalcin, intact Fibroblast growth factor 23 and uncarboxylated-unphosphorylated Matrix-Gla Protein, biomarkers of bone metabolism. A EFLM-IFCC joint study. Osteoporos Int 2020;31:1461-70.

31. Clouet-Foraison N, Marcovina SM, Guerra E, Aarsand AK, Coşkun A, Díaz-Garzón J, et al. Analytical performance specifications for lipoprotein(a), apolipoprotein B-100, and apolipoprotein A-I using the biological variation model in the EuBIVAS population. Clin Chem 2020;66:727-36.

32. Carobene A, Guerra E, Marqués-García F, Boned B, Locatelli M, Coşkun A, et al. Biological variation of morning serum cortisol: updated estimates from the European biological variation study (EuBIVAS) and meta-analysis. Clin Chim Acta 2020;509:268-72.

33. Carobene A, Lao EG, Simon M, Locatelli M, Coşkun A, Díaz-Garzón J, et al. Biological variation of serum insulin: updated estimates from the European Biological Variation Study (EuBIVAS) and meta-analysis. Clin Chem Lab Med 2022;60:518-22.

34. Cavalier E, Fraser CG, Bhattoa HP, Heijboer AC, Makris K, Ulmer $C Z$, et al. Analytical performance specifications for 25-hydroxyvitamin D examinations. Nutrients 2022;60:523-32.

35. Bottani A, Aarsand AK, Banfi G, Locatelli M, Coşkun A, Díaz-Garzón J, et al. European Biological Variation Study (EuBIVAS): within- and between-subject biological variation estimates for serum thyroid biomarkers based on weekly samplings from 91 healthy participants. Clin Chem Lab Med 2022; 60:523-32.

36. Coşkun A, Aarsand AK, Sandberg S, Guerra E, Locatelli M, DíazGarzón J, et al. Within- and between-subject biological variation data for tumor markers based on the European Biological Variation Study. Clin Chem Lab Med 2022;60:543-52.

37. Aarsand AK, Kristoffersen AH, Støve B, Coşkun A, Fernandez-Calle P, Díaz-Garzón J, et al. Bayesian based biological variation data for coagulation markers from the EuBIVAS. Clin Chem 13 Aug 2021. https://doi.org/10.1093/clinchem/hvab100. [Epub ahead of print].

38. Roraas T, Stove B, Petersen PH, Sandberg S. Biological variation: the effect of different distributions on estimated within-person variation and reference change values. Clin Chem 2016;62:725-36.

39. Kristoffersen AH, Petersen PH, Røraas T, Sandberg S. Estimates of within-subject biological variation of protein $\mathrm{C}$, antithrombin, 
protein S free, protein S activity, and activated protein C Resistance in pregnant women. Clin Chem 2017;63:898-907.

40. Røraas T, Sandberg S, Aarsand AK, Støve B. A Bayesian approach to biological variation analysis. Clin Chem 2019;65: 995-1005.

41. Bartlett WA, Braga F, Carobene A, Coşkun A, Prusa R, FernandezCalle $P$, et al. Biological variation working group, European federation of clinical chemistry and laboratory medicine (EFLM). A checklist for critical appraisal of studies of biological variation. Clin Chem Lab Med 2015;53:879-85.

42. Carobene A. The European biological variation study (EuBIVAS): delivery of updated biological variation estimates, a project by the working group on biological variation in the European federation of clinical chemistry and laboratory medicine. J Lab Precis Med 2017;2:70.

43. Røraas T, Petersen PH, Sandberg S. Confidence intervals and power calculations for within-person biological variation: effect of analytical imprecision, number of replicates, number of samples, and number of individuals. Clin Chem 2012;58: 1306-13.

44. Jonker N, Aslan B, Boned B, Marqués-García F, Ricós C, Alvarez $V$, et al. Critical appraisal and meta-analysis of biological variation estimates for kidney related analytes. Clin Chem Lab Med 2022;60:469-78.

45. Díaz-Garzón J, Fernández-Calle P, Minchinela J, Aarsand AK, Bartlett WA, Aslan B, et al. Biological variation data for lipid cardiovascular risk assessment biomarkers. A systematic review applying the biological variation data critical appraisal checklist (BIVAC). Clin Chim Acta 2019;495:467-75.

46. González-Lao E, Corte Z, Simón M, Ricós C, Coskun A, Braga F, et al. Systematic review of the biological variation data for diabetes related analytes. Clin Chim Acta 2019;488:61-7.

47. Diaz-Garzon J, Fernandez-Calle P, Sandberg S, Ozcurumez M, Bartlett WA, Coskun A, et al. Biological variation of cardiac troponins in health and disease: a systematic review and metaanalysis. Clin Chem 2021;67:256-64.

48. Marqués-García F, Boned B, González-Lao E, Braga F, Carobene A, Coskun A, et al. Critical review and meta-analysis of biological variation estimates for tumor markers. Clin Chem Lab Med 2022; 60;494-504.

49. Ceriotti F, Fernandez-Calle P, Klee GG, Nordin G, Sandberg S, Streichert T, et al. Criteria for assigning laboratory measurands to models for analytical performance specifications defined in the 1st EFLM Strategic Conference. Clin Chem Lab Med 2017;55:189-94.

50. Braga F, Panteghini M. Derivation of performance specifications for uncertainty of serum C-reactive protein measurement according to the Milan model 3 (state of the art). Clin Chem Lab Med 2020;58:e263-5.

51. Fraser CG. Biological variation: a still maturing aspect of laboratory medicine. Adv Lab Med 2019;1:20190032.

Supplementary Material: The online version of this article offers supplementary material (https://doi.org/10.1515/cclm-2021-0370). 